\title{
EL PAPEL DE LA COMUNICACIÓN EN LA CONSTRUCCIÓN NACIONAL DE CATALUÑA: \\ JORDI PUJOL Y LA INSTRUMENTALIZACIÓN POLÍTICA \\ DE LOS MEDIOS (1968-1989)
}

\section{The role of communication in Catalonia's nation building: Jordi Pujol and the political instrumentalization of the media (1968-1989)}

\author{
JOSEP ÀNGEL GUIMERÀ I ORTS \\ Universitat Autònoma de Barcelona \\ Josepangel.guimera@uab.cat
}

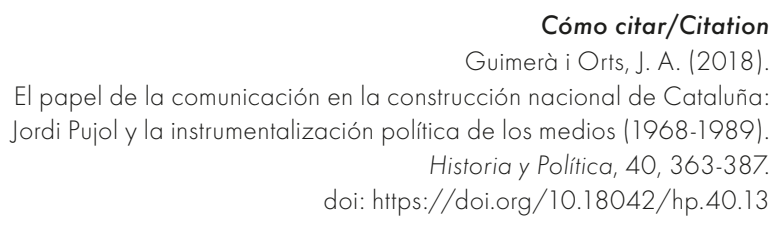

(Recepción: 06/04/2017. Evaluación: 18/07/2017. Aceptación: 01/12/2017. Publicación: 02/11/2018)

\section{Resumen}

Desde el inicio de su actividad política bajo el franquismo y durante los veintitrés años que presidió la Generalitat de Catalunya, Jordi Pujol mostró un gran interés per intervenir en los medios de comunicación. Este artículo tiene por objetivo describir e interpretar las acciones llevadas a cabo por Pujol para crear y/o controlar medios que le ayudasen a conseguir sus objetivos políticos durante su etapa de opositor antifranquista y sus dos primeras legislaturas como presidente de la Generalitat. Para conseguir estos objetivos se diseńó una estrategia de investigación cualitativa que combina fundamentos teóricos de la ciencia política y las ciencias de la comunicación y que triangula las técnicas del análisis documental (marco legal, literatura gris, monografías y artículos científicos, libros de memorias) y las entrevistas en 
profundidad a los protagonistas de las acciones analizadas, incluido el propio Jordi Pujol. El resultado es una investigación original que aporta luz sobre las relaciones entre movimientos políticos y medios de comunicación en un momento fundacional como la Transición española. El artículo muestra una clara voluntad intervencionista de Pujol sobre unos medios que consideraba infiltrados por partidos de izquierda y hostiles al nacionalismo catalán. Siempre tuvo una concepción instrumental del periodismo, tanto para la promoción de su partido e ideas políticas como en el proceso de (re)construcción nacional de Cataluña (proyecto compartido con otras formaciones). De acuerdo con los resultados obtenidos, Pujol convirtió la creación de medios de comunicación en un objetivo fundamental de su acción política.

\section{Palabras clave}

Nacionalismo; medios de comunicación; Cataluña; Jordi Pujol; comunicación política.

\section{Abstract}

From the beginning of his political activity under the Franco regime and during the 23 years of his presidency of the Generalitat de Catalunya, Jordi Pujol showed a great interest to intervene in the media. This article aims to describe and interpret the actions carried out by Pujol to create and/or control the media in order to help them achieve their political objectives during his anti-Francoist opposition activities and his first two legislatures as president of the Generalitat. To achieve these objectives, a qualitative research strategy was designed. It combines theoretical foundations of political science and communication sciences and triangulates the techniques of documentary analysis (legal framework, gray literature, monographs and scientific articles, and memoirs) and in-depth interviews with the protagonists of the actions analyzed, including Jordi Pujol himself. The result is an original research that sheds light on the relations between political movements and the media during the Spanish transition, a foundational moment in Spanish politics. The article shows a clear interventionist will of Pujol on the media, considered by him as infiltrated by leftist parties and hostile to Catalan nationalism. He always had an instrumental conception of journalism, both for the promotion of his party and political ideas and in the process of national (re)construction of Catalonia (a project that he shared with other parties). According to the results obtained, Pujol made the creation of mass media a fundamental objective of his political action.

\section{Keywords}

Nationalism; mass media; Catalonia; Jordi Pujol; political communication. 


\section{SUMARIO}

I. INTRODUCCIÓN. II. JORDI PUJOL Y LA CREACIÓN E IMPULSO DE MEDIOS DE COMUNICACIÓN EN CATALUÑA: 1. Un activista antifranquista que quería editar prensa catalanista. 2. Un proyecto multimedia de la prensa afín. 3. La CCRTV, o la piedra de toque del proyecto pujolista. 4. Las subvenciones: entre las ayudas al catalán y el apoyo al catalanismo. 5. Un sistema de comunicación catalán con medios catalanistas. III. CONCLUSIONES. Bibliografía.

\section{INTRODUCCIÓN'}

A mediados de los años sesenta, después de pasar dos años en prisión, Jordi Pujol dejaba atrás una etapa de activismo antifranquista de fuerte carácter cultural e iniciaba un periodo de mayor actividad política clandestina desde posiciones nacionalistas. En esos años, Pujol diseńó el que sería su proyecto político a largo plazo y que denominaría "Construir Catalunya». Consistía en dos grandes líneas de actuación fuertemente interrelacionadas. Por una parte, recuperar, consolidar y en la medida de lo posible ampliar el autogobierno perdido por Cataluña bajo el franquismo. Por otro, contribuir a la recuperación de la identidad nacional catalana y, en especial, de su principal seña: el catalán y la cultura que este vehicula ${ }^{2}$.

Dentro de este proyecto, los medios de comunicación de masas tenían, desde el punto de vista de Pujol, un papel central. Esto le llevó a intervenir sobre el sistema mediático catalán desde el principio. Esta actuación no ha sido menor y se ha desarrollado bajo numerosas y variadas formas a lo largo del tiempo: desde la compra de publicaciones periódicas cuando era un banquero opositor al franquismo en los ańos sesenta a la puesta en marcha de la radio y la televisión de la Generalitat cuando llegó al poder en 1980, pasando por las subvenciones a publicaciones periódicas o la concesión de frecuencias para crear emisoras privadas de radio. De hecho, la articulación de un sistema

1 Este artículo se ha basado parcialmente en el trabajo de campo realizado en el marco de la beca Jordi Pujol que el Centre d'Estudis Jordi Pujol otorgó al autor en 2012. El autor quiere hacer llegar su agradecimiento a los/las revisores/as del original, que con sus comentarios y aportaciones han mejorado claramente la versión publicada.

2 Culla (2001); Guibernau (2002); Crameri (2008); Pujol (2009); Dowling (2013), y Amat (2015). 
mediático catalán potente, en catalán y lo más autónomo posible, fue uno de los objetivos a largo plazo que los primeros Gobiernos de Pujol persiguieron con más ahínco ${ }^{3}$.

La investigación en la que se basa este texto tiene por objeto de estudio las acciones llevadas a cabo por Jordi Pujol, tanto desde el ámbito privado como desde el Gobierno de la Generalitat, para crear y/o controlar medios de comunicación en Cataluña que le ayudasen a llevar a cabo sus objetivos políticos. Por objetivos políticos entendemos tanto la consecución y/o mantenimiento del poder como aquellos relacionados con su proyecto de construcción nacional. En relación con este objeto, se plantean dos objetivos específicos. Por una parte, identificar qué papel atribuía Pujol a los medios en su proyecto para Cataluña. Se trata de explorar qué concepción tenía de ellos y cómo este punto de vista guio su intervención. Este aspecto es clave porque, como reconoce él mismo, fue un "presidente presidencialista» ${ }^{4}$. Por otra parte, describir $\mathrm{y}$ analizar las estrategias desplegadas para crear esos medios.

Para esta investigación se ha delimitado un periodo temporal de veintiún años establecido a partir de la identificación de acciones significativas de Pujol en el sector de la comunicación. El punto de inicio del periodo es la entrada de Pujol por primera vez en el capital de un medio privado (El Correo Catalán, en 1968). El punto de cierre es la creación del segundo canal de televisión de la Generalitat (Canal 33, en 1989), que él mismo considera un hito del nacionalismo catalán $n^{5}$. De esta forma, el análisis incluye dos etapas muy diferentes en la carrera de Pujol. Una primera formada por sus primeros años como político antifranquista tras una etapa de agitador cultural nacionalista. Y una segunda que incluye las dos primeras legislaturas de $\mathrm{CiU}$ al frente del Gobierno de la Generalitat recuperada. Sobre el cierre temporal de la investigación en 1989, cabe destacar que existe un amplio consenso académico ${ }^{6}$ sobre que durante los primeros ocho años de ejecutivos convergentes se conformó un manera de construir administración y de gobernar que marcaría las décadas siguientes, incluso más allá de los veintitrés años de "pujolismo». Esto es muy claro en el ámbito mediático, tal y como hemos puesto de manifiesto en otras

Martínez y Oliveres (2005); Lo Cascio (2008); Pujol (2009), y Guimerà (2014).

4 Pujol (2009): 183. En este sentido, cabe destacar que, en 2017, existen escasos estudios científicos sobre el pensamiento de Pujol sobre los medios. A pesar de ser considerado uno de los principales ideólogos del catalanismo contemporáneo por autores como Culla (2001) o Guibernau (2002), no se le conocía un discurso explícito sobre la comunicación de masas, como evidenció Guimerà (2014).

Comunicación personal, 5 de septiembre de 2013.

6 Culla (2001); Lo Cascio (2008); Crameri (2008), y Dowling (2013). 
investigaciones ${ }^{7}$. Siguiendo a $\mathrm{Amat}^{8}$, la actividad parapolítica bajo el franquismo a partir de finales de los sesenta y la presidencia de la Generalitat de Catalunya —especialmente, los primeros ańos- son dos variantes metodológicas para el gran objetivo político de Pujol: la nacionalización de la sociedad catalana. Los ańos considerados, pues, permiten observar dos maneras de abordar el problema por parte del líder nacionalista catalán.

Ciertamente, esta investigación no es el primera que aborda las complejas relaciones de Pujol con los medios. Existe en Cataluña una larga línea de aproximaciones —que algunos autores denominan «escuela catalana de comunicación»- que ha tenido en la configuración de un sistema mediático catalán uno de sus principales centros de interés ${ }^{9}$. Pero como ponen de manifiesto esos mismos investigadores, se trata de trabajos mayoritariamente descriptivos que se centran en la relación entre los medios y el proceso de normalización lingüística y cultural —en especial, la evolución de la oferta en catalán-. Solo algunas aproximaciones buscaban profundizar en las variadas e intrincadas relaciones entre partidos, Gobiernos y comunicación en la Cataluña autonómica desde una perspectiva política, pero solo a partir de los años noventa ${ }^{10}$. Este artículo quiere ayudar a ampliar el conocimiento sobre este aspecto, poco tratado en trabajos previos - especialmente en el periodo del tardofranquismo y primeros años de democracia-.

Así pues, el problema de investigación que se aborda aquí es el poco conocimiento existente y la escasa teorización sobre cómo nacieron y cómo evolucionaron con el fin del franquismo la relaciones entre medios de comunicación y movimientos políticos democráticos —en concreto, el nacionalismo catalán impulsado por Pujol— en un periodo histórico seminal como es la transición española a la democracia. Los primeros indicios y evidencias recogidos en el trabajo de campo apuntaban con claridad a una concepción instrumental de los medios por parte de Pujol, ya que los entendía como herramientas tanto para dar a conocer su ideología política como para llevar a cabo su proyecto de construir Cataluña desde posiciones nacionalistas ${ }^{11}$. De acuerdo con estas primeras observaciones se decidió acudir, para guiar esta investigación, a un marco teórico que pudiera dar cuenta de unas interacciones entre los sistemas político y mediático que en Cataluña aparecían como altamente complejas. Así, se optó por combinar aportaciones teóricas de

Guimerà (2013 y 2014).

Amat (2015): 288.

Corominas (1997); Jones (1998), y Guimerà (2002 y 2014).

Guillamet (1996); Fernández y Blasco (2005); Jones (2007), y Guimerà (2014).

11 Antich (1994); Jones (2007), y Pujol (2007 y 2009). 
procedencias y alcances diferentes para poder formular preguntas de investigación pertinentes que delimitaran el problema y ayudaran a dar cuenta de él de manera concreta.

Si en la última década hay una aproximación teórica que sea referencia en el análisis de las interacciones entre los sistemas político y mediático, esta es la desarrollada por los politólogos Daniel C. Hallin y Paolo Mancini ${ }^{12}$. En su obra identifican tres grandes modelos de sistemas de medios: el modelo del Atlántico norte o liberal; el del norte y centro de Europa o democrático corporativo; y el mediterráneo o pluralista polarizado, en el cual se encuentra España. Una de las características del modelo Mediterráneo es la «instrumentalización», que definen como "la utilización de los medios de comunicación por parte de varios actores como instrumentos para intervenir en el mundo político» ${ }^{13}$. Entre estos actores incluyen desde los Gobiernos a los partidos políticos pasando por la Iglesia o los empresarios de la comunicación. Para estos autores, esto se debe a que en los países del sur de Europa aún perdura la concepción con la que nacieron los periódicos de masas en esta zona a finales del siglo xix: como una extensión de la política e incluso de los partidos ${ }^{14}$. De acuerdo con esto, los medios se entienden como un «medio de expresión ideológica y de movilización política» y no tanto como agentes informativos. Sobre esta base apoyamos la primera pregunta de investigación: ¿ ¿La intervención de Jordi Pujol en la adquisición y/o puesta en marcha de medios de comunicación, públicos y privados, era de carácter instrumental?

Si bien es cierto que el concepto de instrumentalización pudiera parecer suficiente para explicar el comportamiento de Pujol, la politóloga Montserrat Guibernau apunta que los movimientos políticos nacionalistas de naciones sin Estado como Cataluña tienen una relación peculiar con los medios de comunicación — que iría más allá de la apuntada de forma genérica por Hallin y Mancini- ${ }^{15}$. Es por esta razón que hemos acudido a Guibernau y sus aportaciones sobre el «nacionalismo de las naciones sin Estado», o «nacionalismo de oposición» — del cual es un exponente claro el catalanismo de Pujol— ${ }^{16}$. Guibernau dibuja a los nacionalismos de oposición como unos movimientos políticos enfrentados con el Estado dentro del cual actúan, que a su vez elabora y despliega un nacionalismo propio, que denomina «estatal ${ }^{17}$. En este conflicto,

\footnotetext{
Hallin y Mancini (2004).

Hallin y Mancini (2004): 34 y 105.

Hallin y Mancini (2004): 34.

Guibernau (1999 y 2002).

Guibernau (2002): 59 y ss.

17 Guibernau (1999): 59 y 104-105.
} 
los nacionalistas de oposición suelen tener limitado el acceso a los medios de comunicación. Si ese movimiento está proscrito — como ocurría bajo el franquismo-, el acceso está directamente prohibido. Ante esta circunstancia, «las minorías nacionales intentan crear sus propios canales de comunicación, que siempre están amenazados», para "poder exponer sus causas concretas».

La autora también sostiene que, cuando «a la nación minoritaria se le ha garantizado algún tipo de autonomía política dentro del Estado», se le permite la creación de medios propios. En este contexto, los líderes del nacionalismo no estatal los necesitan para convencer a la población de la necesidad de subscribirse a sus programas políticos y para cuestionar al Estado central. También los necesitan para transmitir una imagen moderna y democrática, ya que los nacionalistas de oposición suelen ser presentados de forma muy negativa en los medios del Estado. De acuerdo con todo esto, pues, las élites nacionalistas tendrían un interés claro por crear medios. Esto atribuye al catalanismo un perfil específico que iría más allá que la instrumentalización definida por Hallin y Mancini. Basándonos en los presupuestos teóricos de Guibernau se ha formulado una segunda pregunta de investigación: si la concepción de los medios por parte de Pujol era instrumental, ¿atendía a los intereses y necesidades específicos del movimiento nacionalista que lideraba o iban más allá?

El conocimiento científico disponible sobre Cataluña apunta claramente a que los medios de comunicación han sido considerados, por actores de todo tipo, como unos agentes básicos para la normalización y extensión del uso de las lenguas menos habladas y/o perseguidas; y también para la (re)creación y refuerzo de identidades nacionales en una sociedad cada vez más globalizada. Según sostiene uno de los principales autores catalanes en este campo, Josep Gifreu, una nación que quiera asegurar su continuidad en el tiempo necesita un sistema comunicativo propio: «Hoy resulta imposible la afirmación de la diferencia y de la identidad sin disponer de una amplia capacidad de control del espacio de comunicación propio o sin una presencia importante en los mass media y en la cultura de masas $»^{18}$. Acudiendo de nuevo a Guibernau, la necesidad de estos medios se enmarca en la lucha política entre nacionalismo de oposición y nacionalismo de Estado. Para esta autora, la comunicación de masas ha sido uno de los instrumentos — junto al sistema educativo- del cual se han dotado los Estados para tratar de homogeneizar culturalmente a la población bajo su poder, reduciendo así la diversidad nacional existente en su interior ${ }^{19}$.

18 Gifreu (1991): 254.

19 Guibernau (2002 y 2010). 
Según Guibernau, la identidad nacional es un elemento de legitimación política de los Estados nación contemporáneos, que la promueven a través del nacionalismo porque la consideran un vehículo de cohesión social y una generadora de lealtad hacia el propio estado - que se aseguraría, así, su propia continuidad a largo plazo- . En el planteamiento de esta autora, los medios de comunicación y el sistema educativo son usados por el Estado nación para «la propagación de una "imagen de la nación” particular, con sus símbolos y rituales, sus valores y principios, sus tradiciones, sus estilos de vida, sus enemigos comunes y, una cosa aún más importante, una clara definición de cómo ser un "buen ciudadano" $»^{20}$.

Para Guibernau, a partir de 1980 la Generalitat presidida por Pujol funcionó como un contrapoder que se opuso a esa homogeneización cultural promoviendo sus propias políticas de identidad para asegurar la continuidad nacional de Cataluña ${ }^{21}$. Así, las competencias obtenidas por la Generalitat a través de la Constitución y del Estatuto de Autonomía la convirtieron en un "casi-Estado» que desplegó su propio nacionalismo tanto para cohesionar a la sociedad bajo su administración como para legitimarse a ella misma a largo plazo. De acuerdo con esto, los medios son instrumentos usados por dos nacionalismos enfrentados que intentan (re)construir dos naciones distintas en el mismo espacio y tiempo.

A partir de este planteamiento surge nuestra tercera pregunta de investigación: dentro de este enfrentamiento entre Generalitat y Estado, ¿qué papel juegan los medios de comunicación en el refuerzo de la identidad nacional catalana dentro de las políticas de Jordi Pujol? A las tres preguntas formuladas hasta ahora, añadimos una cuarta orientada a conocer el cómo de todo este proceso: ¿cuáles fueron las estrategias utilizadas por Pujol para crear esos medios para superar las limitaciones que suelen afectar a los medios de las naciones sin Estado y poner en marcha su proyecto de construcción nacional?

De acuerdo con el marco teórico y los objetivos de conocimiento establecidos, el diseño metodológico fue cualitativo. Como sostiene Soriano, estos diseños están orientados a la generación de conocimiento nuevo y permiten captar mejor la complejidad de fenómenos poliédricos como el problema de investigación tratado aquí ${ }^{22}$. En coherencia con este planteamiento, se usaron dos técnicas de investigación que permitieran la triangulación de resultados. La primera fue el análisis documental, que se aplicó con exhaustividad sobre las normas legales que rigieron las políticas de la Generalitat durante los años

\footnotetext{
20 Guibernau (2010): 60-61.

21 Guibernau (2002): 28, 64-65 y 126-127.

22 Soriano (2007).
} 
estudiados. También se han analizado discursos, conferencias y obras de pensamiento de Jordi Pujol. Han resultado especialmente importantes los volúmenes primero y segundo de sus memorias, publicados en 2007 y 2009 . A su vez, se han analizado monografías de corte histórico, la mayoría elaboradas por periodistas, que han descrito el paso de Pujol por empresas de comunicación privadas durante el periodo estudiado ${ }^{23}$. La segunda fue la entrevista en profundidad. Se entrevistó a once altos cargos de Gobiernos catalanes entre 1980 y 2003 que trabajaron en el diseño de las políticas de medios. Para esta investigación han sido clave las dos realizadas a Jordi Pujol.

\section{JORDI PUJOL Y LA CREACIÓN E IMPULSO DE MEDIOS DE COMUNICACIÓN EN CATALUÑA}

La información recogida durante el trabajo de campo señala que Jordi Pujol desarrolló, entre 1968 y 1989, una fuerte actividad orientada a la creación y/o impulso de medios de comunicación de alcance autonómico que tuvieran una fuerte capacidad de influencia sobre la sociedad. Las estrategias, que se describirán a continuación, fueron cuatro: adquisición de cabeceras de prensa, creación de la radiotelevisión pública autonómica, creación de un grupo multimedia privado y la concesión de subvenciones por parte de la Generalitat a publicaciones impresas. Las razones apuntadas para legitimar cada una de estas acciones también son complejas y muestran claramente el carácter poliédrico de la concepción que Pujol tenía de los medios de comunicación.

\section{UN ACTIVISTA ANTIFRANQUISTA QUE QUERÍA EDITAR PRENSA CATALANISTA}

Desde muy joven, Pujol se implicó en entidades culturales y cívicas catalanistas que se oponían tanto al carácter dictatorial del régimen franquista como, en particular, a la persecución de la cultura y la lengua catalanas. Pero a mediados de los años sesenta, su activismo cambió y avanzó hacia acciones de carácter parapolítico, previendo un futuro cambio de régimen y la necesidad de recuperar las instituciones de autogobierno catalán en una España democrática ${ }^{24}$. Pujol llevó a cabo esta actividad clandestina desde dos

23 Faulí et al. (1987); Huertas (2005); Geli y Huertas (1990), y Saura (1998).

24 Pujol (2007); Antich (1994); Dowling (2013), y Amat (2015). 
entidades muy diferentes: Banca Catalana y el parapolítico Centre d'Informació, Recerca i Promoció (CIRP). El primero era el banco que había cofundado en 1959 y que convertiría en un instrumento de intervención política. El CIRP era, según explica el mismo Pujol ${ }^{25}$, un ente sustitutivo del partido que hacía falta para construir Catalunya pero que la dictadura impedía crear. A través de estas dos entidades, y también con recursos económicos propios, decidió entrar en el sector de la prensa a finales de los años sesenta.

En 1968 compró las primeras acciones de El Correo Catalán. En 1974, el mismo año en que cofundó Convergència Democràtica de Catalunya (CDC), pasaría a formar parte del Consejo de Administración. Cuando entró en la empresa, esta estaba en manos de la burguesía textil catalana, pero parecía decidida a vendérsela a la familia del entonces alcalde de Barcelona, José María Porcioles. Como el Ministerio de Información y Turismo, que debía autorizar los cambios en la propiedad, hizo un informe negativo por sus antecedentes penales, Pujol se sirvió de fiduciarios para controlar la empresa. De hecho, la propiedad del medio en la época que estuvo controlado por él nunca fue clara del todo. Pujol sostuvo durante años que había desinvertido al ser elegido diputado de las Cortes en 1977. Solo mucho tiempo después, cuando publicó sus memorias, se supo que había sido propietario mayoritario hasta 1986, año en que la empresa fue liquidada después de cuantiosas pérdidas y cuando Pujol ya llevaba seis años como presidente ${ }^{26}$.

Según Saura, la intención de Pujol era contar con un medio afín, tal como ocurría con la prensa de partido antes de la Guerra Civil. Pujol intervenía activamente en la línea editorial del periódico, con presencia «incluso física» constante, ya que entendía la participación en El Correo como una inversión y quería resultados políticos. Martínez y Oliveres sostienen que Pujol no se limitaba a la gerencia, sino que llegó a redactar editoriales e intentaba marcar la línea informativa. Introdujo redactores afines y todo ello llevó a no pocos conflictos con los trabajadores históricos del medio, que trataban de resistirse - en grados diversos - a actuaciones de los hombres de Pujol como decidir la portada, modificar titulares o tratar positivamente al político y a su partido ${ }^{27}$.

También en 1974 Pujol se hizo con el control de la prestigiosa revista Destino, aunque sin constar en el proceso de compra, que se hizo de nuevo a través de fiduciarios. En esta ocasión, su propietario, Josep Vergés, se la ofreció cansado de problemas económicos y políticos. Se hacía así con el control de una

\footnotetext{
25 Pujol (2007): 184-186.

26 Martínez y Oliveres (2005): 153-154, y Pujol (2007): 259-260.

27 Saura (1998): 23-24 y 98, y Martínez y Oliveres (2005): 157.
} 
publicación de referencia para ciertos sectores intelectuales que valoraban los artículos de destacados colaboradores como Josep Pla. Según Geli y Huertas, la adquisición se debe entender, como en el caso de El Correo Catalán, dentro de los cambios en el marco político y en la idea de Pujol de que «disponer de una prensa propia que le ayudaría en sus planes». Con la entrada de Pujol, la revista hizo una declaración conceptual de lo que debía ser a partir de ese momento: "Cabía una adaptación a la nueva realidad política y social más viva y abierta al futuro, la defensa de la libertad política, del progreso y de la democracia social, además de una inequívoca afirmación de catalanidad». A partir de entonces, serían habituales los elogios (en ocasiones formulados «ardientemente») de los actos de Jordi Pujol, las participaciones como autores invitados de miembros del partido próximos a él o las críticas a los partidos de izquierdas. Todo esto provocó de nuevo tensiones con la redacción, con constantes destituciones y dimisiones hasta que Pujol se deshizo de Destino en $1978^{28}$.

A lo largo de los setenta, Pujol también participó en la gestión de las revistas Oriflama y Canigó $^{29}$. A finales de los sesenta eran vistas como las revistas más representativas de un nuevo periodismo, que trataba de ofrecen información libre, catalana y en catalán ${ }^{30}$. La primera era una publicación juvenil catalanista creada por el Obispado de Vic en 1960. Pujol ayudó desde muy pronto a su financiación a través de Banca Catalana y de sus propios recursos personales. En 1970, una deficitaria Oriflama ya dependía financieramente del político, que incidía habitualmente en su gestión y en los contenidos, especialmente los políticos. De nuevo, la introducción de afines en el Consejo de Administración y en la redacción fue la estrategia seguida. De nuevo, los conflictos con la redacción y con los responsables históricos fueron moneda corriente, sobre todo en relación a la incorporación o despido de colaboradores en función de su ideología. También por las negativas del Consejo de Redacción a publicar artículos de Pujol, que este enviaba bajo pseudónimo. El político abandonó la revista en 1972, que desaparecería en $1977^{31}$.

Canigó surgió en 1954 como una revista innovadora y abierta que progresivamente había ido ampliando los textos en catalán. Pujol la financió con inversiones a fondo perdido y publicidad de Banca Catalana y en 1970 intentó hacerse con el control. Según cuentan el editor y la directora en ese momento, en 1971 Pujol les hizo saber que la ayuda no era desinteresada, sino que había

\footnotetext{
28 Geli y Huertas (1990): 69, y Dowling (2013): 235.

29 Martínez y Oliveres (2005): 159-160; Pujol (2007): 256 y ss., y Faulí et al. (1987): 60-61.

30 Lo Cascio et al. (2005): 127.

31 Faulí et al. (1987): 60-61 y 64.
} 
consistido en una compra a plazos. De acuerdo con esto, Pujol se consideraba propietario y exigió el control de la cabecera. No lo consiguió y retiró su apoyo, hecho que debilitó a la publicación, que sobreviviría hasta $1983^{32}$.

La intervención de Pujol en estas publicaciones de difusión limitada —alguna de ellas de alcance local— se debe entender en el marco de su papel cualitativamente importante en los campos cultural e intelectual en la Cataluña del tardofranquismo. Algunas con larga tradición a sus espaldas, todas destacaron por un cierto papel de vehiculización de ideas contrarias al franquismo y, a su vez, promotoras de la cultura catalana. También contaban en su haber con enfrentamientos de grado diverso con la censura franquista, hecho que les daba una pátina democrática muy potente ante la sociedad catalana. Como explica Lo Cascio, «las publicaciones intelectuales más minoritarias tenían más posibilidades de ofrecer artículos que no habrían visto la luz en periódicos de mayor tirada ${ }^{33}$. Para el Pujol conspirador contra el franquismo, esto aparecía como una ventaja competitiva muy atractiva.

Por su parte, Amat argumenta que la entrada en todos estos medios se produce en un momento muy particular: cuando Pujol plantea una batalla por la hegemonía dentro del catalanismo justo en el momento en el que este se reconstruía tras el desastre de la Guerra Civil ${ }^{34}$. En el tardofranquismo, la concepción dominante del catalanismo era la progresista, con una fuerte declinación marxista a partir de los primeros años setenta. Pujol, que considera el marxismo uno de los principales frenos para su proyecto nacionalizador, decide plantear una batalla de las ideas para convertir en hegemónica su visión más nacionalista y conservadora del catalanismo ${ }^{35}$. En este combate, que se entremezcla con su construcción como líder político, los medios de comunicación aparecían como imprescindibles para diseminar su propuesta ideológica.

Cuando se le pregunta a Pujol por qué hizo estas inversiones, aparece claramente explicitada su voluntad de influir en la sociedad en un momento en el cual se intuye un cambio de régimen y en el cual él mismo quiere participar. En una de las entrevistas para esta investigación, el expresidente explica que quería "difundir ideología» a través tanto de "revistas de pensamiento» minoritarias (Destino, Oriflama, Canigó) como a través de medios que permitieran hacer circular «mensajes masivos» (El Correo Catalán) ${ }^{36}$. En sus memorias, Pujol explica que «el fin del franquismo se entreveía y teníamos que

\footnotetext{
Faulí et al. (1987): 88-89, y Lo Cascio et al. (2005): 127.

Lo Cascio (2005): 116.

Amat (2015): 320-325.

Amat (2015); Crameri (2008), y Dowling (2013).

36 Comunicación personal, 5 de septiembre de 2013.
} 
conseguir que la prensa estuviera al servicio de la democracia y el catalanismo». Sobre la compra de El Correo Catalán sostiene que la razón para comprarlo era que "podía ser útil para la difusión de un catalanismo más moderno, más de centroizquierda».

Sobre este aspecto, Pujol es muy claro: «Me acusaron de querer influir en los medios de los cuales fui propietario. Un empresario de prensa, si no se trata de un caso extraño, que no digo que no exista, siempre quiere influir de alguna manera, sea políticamente, económicamente o socialmente. Si no, no haría prensa, haría otra cosa». Ahora bien, Pujol también reconoce que su estrategia fue un fracaso y se muestra muy crítico con su etapa de editor, ya que quedó «muy mal con mucha gente» y no consiguió sus objetivos: «Nos costaba mucho introducir la ideología que nosotros queríamos. El nacionalismo estaba muy mal visto, entre los periodistas y los intelectuales de aquel momento. Predominaban ideas muy de izquierdas ${ }^{37}$.

\section{UN PROYECTO MULTIMEDIA DE LA PRENSA AFÍN}

Con la llegada al poder en 1980, Pujol también utilizó los recursos políticos y económicos de la Generalitat para ayudar a la creación de medios privados. Durante el periodo estudiado, el principal proyecto en este campo fue la creación de un grupo multimedia regional alrededor de la Fundació Catalana de Comunicació (FCC) ${ }^{38}$.

El proyecto nació a principios de los ochenta impulsado desde las empresas editoras de El Correo Catalán y Avui, primer diario en catalán aparecido después del franquismo e impulsado desde sectores del catalanismo cultural y político. En ese momento, ambas estaban controladas por personas afines a Pujol o CDC. Además, también abarcaba prensa comarcal como El 3 de vuit (Vilafranca del Penedès), La Veu del Maresme (Mataró), Plaça Gran (Granollers) y Ausona (Vic). A partir de 1983 también integró a la cadena privada autonómica Cadena 13, surgida de las concesiones de FM que el Gobierno catalán hizo ese mismo año. A largo plazo, se preveía que también explotara una televisión privada a través de Promotora Catalana de Ràdio i Televisió, aunque este proyecto nunca se concretó. Según Pujol, el proyecto también

Pujol (2007): 256-257.

38 El proyecto acabó siendo un fracaso que se fue consumando por fases. El Correo Catalán desaparecía en 1986 asfixiado por grandes problemas económicos. En 1991 era Cadena 13 la que lo hacía, ahogada también por fuertes deudas. Bonet (1994); Guillamet (1996). 
incluía la conversión de El Correo Catalán en una publicación en catalán que ayudara a la normalización lingüística ${ }^{39}$.

Según explica Jaume Guillamet, con la Fundación «se diseñó desde el Palau de la Generalitat una ambiciosa operación de apoyo a los medios de comunicación nacionalistas», que se basaba en la «obtención de financiación pública y privada» y en «la creación de servicios conjuntos». En este sentido, la concesión de frecuencias a El Correo Catalán y el Avui se explicó desde el Gobierno como parte de su idea de ayudar a los editores a hacer frente a la caída de la difusión ayudándoles en su diversificación multimedia. Pero las concesiones también generaron acusaciones de clientelismo, ya que la oposición en el Parlament de Catalunya consideraba que se quería crear una radio afín al Gobierno: en los consejos de administración se sentaban conocidos miembros de CDC y años después se supo que Pujol seguía controlando $E l$ Correo Catalán cuando se articuló esta operación ${ }^{40}$.

En una entrevista para esta investigación, Pujol afirma que todos los medios que se ayudó a crear desde sus Gobiernos, fueran públicos o privados, tenían «una mirada nacional» y eran "catalanistas» ${ }^{41}$. En este sentido, Pujol siempre mostró su preocupación por la poca presencia mediática tanto de las señas de identidad nacional (sobre todo, la lengua) como de las posiciones catalanistas, que consideraba infrarrepresentadas en los medios informativos en relación con su peso en la sociedad. De hecho, Pujol ha tenido históricamente una tensa relación con los periodistas ${ }^{42}$. Por una parte, y como ya se ha apuntado, porque los consideraba «muy escorados a la izquierda» — sobre todo, durante la Transición - y muy hostiles al catalanismo en general y a su partido en particular: «El ataque político y periodístico siempre se ha hecho hacia Convergència ${ }^{43}$. Esto le lleva a afirmar —en una entrevista con el autor- que en Cataluña nunca ha hecho falta preocuparse por el pluralismo mediático, ya que "el Gobierno ha sido atacado insistentemente» ${ }^{44}$. Además, siempre ha considerado que los medios de comunicación españoles son, en menor o mayor medida y en referencia a las peticiones de mayor autogobierno, «siempre anticatalanes». En este sentido, los acusa de presentar a los catalanes siempre en términos negativos, como «chantajistas», «insolidarios» $\mathrm{y}$ «victimistas» ${ }^{45}$.

39 Guillamet (1996); Bonet (1994), y Pujol (2009).

40 Bonet (1994); Martínez y Oliveres (2005), y Pujol (2007).

41 Comunicación personal, 10 de Septiembre de 2012.

42 Faulí et al., (1987); Geli y Huertas (1990), y Martínez y Oliveres (2005).

43 Pujol (2009): 111, 118 y 383.

44 Comunicación personal, 5 de septiembre de 2013.

45 Pujol (2012): 15 y 16. 


\section{LA CCRTV, O LA PIEDRA DE TOQUE DEL PROYECTO PUJOLISTA}

Durante las dos primeras legislaturas de gobierno de Jordi Pujol (19801988), la Generalitat creó una de las mayores radiotelevisiones autonómicas de la época. En 1988, la Corporació Catalana de Ràdio i Televisió (CCRTV) gestionaba Catalunya Ràdio (con emisiones regulares desde 1983), la emisora musical RAC105 (1984), TV3 (1984) y la radio especializada Catalunya Música (1987). A finales de 1988 también estaba emitiendo en pruebas la segunda televisión del ente, el Canal 33, que empezaría a emitir regularmente en abril de $1989^{46}$.

Tanto Catalunya Radio como TV3 habían nacido como medios generalistas con vocación de ser muy competitivos. Uno de los principales objetivos tanto del Gobierno como de los primeros directivos de la CCRTV fue captar el máximo de audiencia posible a partir de una programación atractiva. Se trataba de evitar, a toda costa, la creación de medios modestos y con una función complementaria que dejaran el protagonismo a medios estatales (RTVE) o privados con sede en Madrid (las principales cadenas radiofónicas del momento). En ningún caso se trataba de crear un canal folklórico, secundario. Si se quería potenciar la presencia del catalán en los medios, esto era clave. En esta línea, la aparición del resto de radios y televisiones de la CCRTV tenía dos objetivos. Por un parte, ampliar la oferta de contenidos de servicio público. Así se descargaba a los canales principales de esta tarea, que podían ofrecer productos más generalistas que captaran audiencias más amplias. Por otra, aumentar la oferta de contenidos en catalán, contribuyendo a una mayor normalización ${ }^{47}$.

Dentro del proyecto de la CCRTV, la joya de la corona era TV3. Pujol entendió muy pronto que la televisión era uno de los principales canales usados por la población para acceder a información y entretenimiento. Asimismo, entendió que era un instrumento de influencia de primer orden. Por eso dedicó esfuerzos y recursos para hacerla una televisión de referencia ${ }^{48}$. Para hacerlo contrató a Alfons Quintà, periodista de El País que había destapado los problemas de Banca Catalana, como director del proyecto. Con esto consiguió un golpe de efecto que le permitía presentar la nueva televisión como un medio autónomo. A su vez, Quintà desarrolló un proyecto moderno de televisión, alejado de la entonces anquilosada RTVE — que aparecía como un antimodelo- ${ }^{49}$. Apostó por los contenidos informativos como columna

\footnotetext{
46 Carreras (1987); Corbella (1991), y Franquet (2001).

47 Lo Cascio (2008); Quintà (1983); Carreras (1987), y Baget (1999).

48 Crameri (2008) y Guimerà (2014).

49 Quintà (1983).
} 
vertebral de la nueva televisión y los rodeó de programas innovadores, series y cine internacionales (sobre todo de EE. UU.). Se contó con asesoramiento de profesionales europeos y estadounidenses, que ayudaron a crear unos programas de gran atractivo ${ }^{50}$.

Para Pujol, que la Generalitat dispusiera de medios potentes era estratégico; tenerlos o no era, de hecho, determinante para su proyecto político para Cataluña. Lo expresó abiertamente en referencia a TV3 en el discurso de investidura de 1980, cuando afirmó que era en el campo de la televisión "donde nos jugamos nuestro ser como catalanes $»^{51}$. En el debate de investidura de 1988 justificó la puesta en marcha del Canal 33 afirmando que «se ha demostrado lo que ya se sabía y, es que en la recuperación lingüística catalana juega un papel decisivo la televisión ${ }^{2}$. En sus memorias, publicadas entre 2007 y 2012, Pujol se ratifica en esta concepción de los medios como instrumentos de normalización lingüística: «En nuestro proyecto de país, una radio y una televisión públicas tenían que ayudar a defender la lengua y a construir la consciencia de catalanidad. [...] Pensaba mucho en la lengua. Me decía a mí mismo: una lengua que no esté presente en los medios de comunicación irá mal ${ }^{53}$. De hecho, Pujol se refiere en diversas ocasiones a la radiotelevisión como «una pieza básica de nuestro proyecto ${ }^{54}$. La CCRTV no dejó de crecer ni en presupuestos ni en oferta mediática durante los veintirés ańos de gobierno ${ }^{55}$, llegando a condicionar en algunos momentos la confección de los presupuestos de la Generalitat, según reconocía el propio Pujol ${ }^{56}$.

Pero la normalización del catalán no fue el único objetivo. Como también reconoce en sus memorias, una de sus intenciones cuando llegó al Gobierno fue «diseminar la conciencia de catalanidad» entre los habitantes de Cataluña, con el objetivo de que todos «fuesen o se sintieran un poco o razonablemente catalanistas o como mínimo identificados con el país». Para Pujol, este proceso era fundamental para asegurar una mínima cohesión social, ya que a finales del franquismo existía una notable fractura entre la población autóctona y la llegada del resto de España. También, para preservar la identidad nacional ${ }^{57}$. Aquí, los medios públicos eran claves: «[...] TV3 no tiene que

\footnotetext{
50 Baget (1999); Quintà (1983), y Crameri (2008).

51 Diari de Sessions del Parlament de Catalunya, núm. 4, de 22 de abril de 1980, p. 23.

52 Diari de Sessions del Parlament de Catalunya, núm. 2, 21 de junio de 1988, p. 12.

53 Pujol (2009): 183.

54 Pujol (2009): 184.

55 Corominas y Moragas (2003) y Guimerà (2014).

56 Comunicación personal, 5 de septiembre de 2013.

57 Pujol (2009): 40.
} 
ser solamente un instrumento lingüístico, en el sentido estricto de la palabra. TV3 tiene que ser un referente, un elemento de identidad y un motivo de orgullo por su calidad. Dicho en lenguaje de siempre: una herramienta de conciencia, de construcción y de identificación del país». En 1988, la creación del Canal 33 se justificaba por la necesidad de reforzar «el sentido de identidad nacional» y "fer país», una expresión que Pujol acuñó en sus años de opositor antifranquista ${ }^{58}$. De hecho, Pujol justificó ante la prensa la creación de la nueva televisión como un buen ejemplo del «nacionalismo activo y responsable» que ejercía su Gobierno.

Como se puede observar, para Pujol el papel de la radiotelevisión pública iba más allá de ser un instrumento lingüístico o cultural. Era, de hecho, un componente básico del concepto mismo de autogobierno y fundamental para su ejercicio efectivo. Así, TV3 y Catalunya Radio reforzaban la existencia de la nación catalana, que legitima el autogobierno y, por lo tanto, la propia existencia de la Generalitat como institución de gobierno. A su vez, con su capacidad de influir sobre la sociedad, los medios públicos dotaban a la Generalitat de poder político efectivo, de capacidad real de intervención sobre la realidad. Para Pujol, si Cataluña quería ser un "país normal», tenía que tener una administración normal ${ }^{59}$. Y en la Europa de los ańos ochenta eso significaba, también, tener medios públicos propios.

El balance que hacía de la CCRTV una vez retirado de la política activa era «muy positivo», ya que había ayudado a «vertebrar el país» transmitiendo «unos valores que le son propios». En una entrevista con el autor, ratificaba esta afirmación sosteniendo que «TV3 ha tenido una orientación catalanista, y esto está muy bien. Es lo que yo quería. Con objetividad y sin falsear las cosas, pero de orientación catalanista $»^{60}$.

\section{LAS SUBVENCIONES: ENTRE LAS AYUDAS AL CATALÁN Y EL APOYO AL CATALANISMO}

En 1983, la Generalitat puso en marcha un sistema de subvenciones a la prensa en catalán pensadas para apoyar a unas publicaciones que sufrían serias limitaciones económicas ${ }^{61}$. Eran ayudas automáticas y se calculaban a partir

\footnotetext{
58 Diari de Sessions del Parlament de Catalunya, núm. 2, 21 de junio de 1988, p. 12.

59 Comunicación personal, 5 de Septiembre de 2013.

60 Pujol (2009): 189, y comunicación personal, 5 de septiembre de 2013.

61 Mateo (1990) y Departament de Cultura (1985 y 1987). En los años 1982 y 1983 ya se habían otorgado algunas ayudas puntuales por el mismo motivo y también a
} 
de cuatro criterios: número de ejemplares difundidos, ámbito de cobertura, número de páginas y periodicidad. Para obtenerlas, las publicaciones tenían que estar editándose en el momento de solicitarlas y se concedían de acuerdo con los datos de difusión auditados por entidades independientes ${ }^{62}$.

Este sistema sigue vigente en 2017 y ha sido fundamental no solo para ayudar al sostenimiento de las publicaciones existentes, sino para ampliar su número, ayudando a configurar el sistema mediático catalán ${ }^{63}$. Durante el periodo considerado aquí, el total de ayudas otorgadas por la Generalitat fue de 4877240 euros, con un total anual creciente que va de los 580233 euros entregados en 1984 a los 788651 distribuidos en 1989 (véase la tabla 1).

TABLA I. Subvenciones a la difusión de prensa periódica en catalán (1984-1989)

\begin{tabular}{cc}
\hline Año & Euros \\
\hline 1984 & $580.233,20$ \\
\hline 1985 & $617.119,85$ \\
\hline 1986 & $656.395,41$ \\
\hline 1987 & $650.243,84$ \\
\hline 1988 & $794.032,44$ \\
\hline 1989 & $788.651,81$ \\
\hline
\end{tabular}

Fuente: elaboración propia a partir de Guimerà (2014: 182).

Los principales beneficiarios eran los diarios y, especialmente, los de alcance autonómico. El decano Avui se benefició ampliamente de este sistema. De hecho, diversas fuentes sostienen que su viabilidad durante esos años y también los posteriores es gracias a las ayudas ${ }^{64}$. Huertas llega a sostener que el periódico, que nació empresarial y financieramente muy débil, no se estabilizó económicamente hasta que Pujol llegó a la presidencia de la Generalitat, momento en el que también el Consejo de Administración pasa

pequeñas publicaciones en castellano. Pero el sistema de ayudas reglado no fue operativo hasta 1984, con la aprobación del decreto 497/1983 (Diari Oficial de la Generalitat de Catalunya, núm. 386, 21 de diciembre de1983).

62 Guimerà (2014).

63 Fernández y Blasco (2005).

64 Guillamet (1996); Jordi Pujol, comunicación personal, 5 de septiembre de 2013; y Lluís Prenafeta, comunicación personal, 13 de septiembre de 2013. 
a ser contralado por miembros de CDC o próximos al partido ${ }^{65}$. Favá, periodista de larga trayectoria en el medio, menciona el dinero no declarado que mandaban Convergència y la Generalitat y que permitía su supervivencia, mientras que al mismo tiempo lamenta la identificación que se hizo del periódico con el partido a pesar de toda la buena voluntad profesional que pudieran poner sus periodistas ${ }^{66}$.

Además de esas ayudas, existen numerosos indicios que apuntan a subvenciones encubiertas y entregadas de manera arbitraria a determinados medios. Según El País, entre 1981 y 1984, el Gobierno había otorgado 3185063,64 euros al Avui, El Correo Catalán, Ràdio Avui-Cadena 13 y la Fundació Catalana de Comunicació. Solo 223 576,50 euros (menos del 10\%) corresponderían a ayudas a la difusión otorgadas con transparencia ${ }^{67}$. Es decir, el Gobierno estaba entregando de manera encubierta subvenciones a medios afines al partido del presidente, y de los cuales él mismo era accionista. En entrevistas con el autor, Pujol reconoció la existencia de ayudas no regladas y también el hecho que al Avui se le otorgara «mucho dinero» ${ }^{68}$. Lo justificaba porque era, hasta la aparición de la edición de El Periódico de Catalunya en 1997, el único diario autonómico en catalán.

En esta línea, quien fuera secretario general de Presidencia de la Generalitat entre 1980 y 1991 y hombre fuerte del Gobierno, Lluís Prenafeta, reconoce en una entrevista para esta investigación que durante los ochenta se otorgaban subvenciones encubiertas a otros medios ${ }^{69}$. Según Prenafeta, uno de los principales beneficiarios de estas ayudas fue La Vanguardia. Como explica Carlos Barrera, durante los años 1983 y 1987 este periódico estuvo dirigido por Francisco Noy, que había formado parte del primer Gobierno de Pujol con un cargo técnico ${ }^{70}$. Según Barrera, llegó a la dirección con el visto bueno de $\mathrm{CiU}$ y como parte de una estrategia de la propiedad del medio para acercarse al nuevo y dinámico Gobierno autonómico en manos nacionalistas.

Ahora bien, cabe matizar que las relaciones entre La Vanguardia y los Gobiernos de Pujol no siempre fueron buenas. El propio Prenafeta acusaba al periódico de españolista y anticatalán - excepto en el periodo en que lo dirigió Noy-, mientras que Pujol se quejaba de que había sido poco afín a sus

65 Huertas (2005): 66-67.

66 Favá (2017): 71.

67 «La Generalitat ha destinado más de 500 millones de pesetas a financiar el grupo periodístico vinculado a Jordi Pujol», El País, 28-04-1984.

68 Comunicación personal, 10 de septiembre de 2012 y 5 de septiembre de 2013.

69 Comunicación personal, 13 de septiembre de 2013.

70 Barrera (1995): 106-107. 
Gobiernos. Eso sí, le reconocía su carácter casi institucional y un papel central en la conformación de la opinión pública. En sus memorias, Pujol sostiene que es muy importante mantener la catalanidad de La Vanguardia y que por eso siempre que ha podido ayudar a esa causa, lo ha hecho ${ }^{71}$.

Barrera aporta un elemento interpretativo clave para entender esta relación: la propiedad de La Vanguardia siempre ha procurado tener buenas relaciones con los poderes políticos municipal, autonómico y central — con los riesgos consecuentes de no satisfacer del todo a ninguno de los tres- Es en este ejercicio de equilibrios que en 1987 sustituiría a Noy como director Juan Tapia, "de conocida afinidad socialista», en una maniobra que Barreda atribuye a la voluntad de Godó de tratar de obtener una concesión de televisión privada que el ejecutivo de Felipe González tenía previsto convocar en los años siguientes ${ }^{72}$. La Vanguardia, pues, siempre fue un medio difícil de controlar por Pujol, hecho que provocó no pocas tensiones.

\section{UN SISTEMA DE COMUNICACIÓN CATALÁN CON MEDIOS CATALANISTAS}

La intervención que los Gobiernos de Jordi Pujol llevaron a cabo sobre el sistema mediático existente en Cataluña no fue la suma de acciones aisladas con objetivos dispersos. Más bien al contrario: Pujol llegó al Gobierno convencido de la importancia de los medios de comunicación no solo para llegar y mantenerse en el poder, sino para recuperar y potenciar tanto la identidad nacional catalana y el autogobierno ejercido a través de la Generalitat. Es este motivo por el que Pujol colocó los medios de comunicación, tanto públicos como privados, en el centro de su acción de gobierno. Crear un sistema de medios de comunicación, lo más completo posible, autocentrado en Cataluña y que defendieran la idea de que esta era una nación, era fundamenta $1^{73}$.

En una entrevista para esta investigación, Pujol afirmaba con rotundidad que, si su Gobierno no hubiera apostado por un sistema mediático propio, «habríamos sido unos ilusos. No nos lo hubiera hecho nadie, a nosotros; no lo habría hecho Madrid ${ }^{74}$. El expresidente defiende que tanto los Estados nación como las naciones sin Estado tienen que invertir «en potenciar y recrear su identidad. Lo hace Francia y lo hace España; y hacen bien». De hecho, para Pujol es evidente que los Gobiernos tienen que invertir «en infraestructuras y

\footnotetext{
Prenafeta (1999) y Pujol (2007).

Barrera (1995): 109.

Pujol (2007); Lo Cascio (2008), y Guimerà (2014).

74 Comunicación personal, 10 de septiembre de 2012.
} 
en bienestar social, pero también en identidad». Es decir, en promover los sentimientos de pertenencia y/o de identificación. Ahora bien, Pujol se quejaba de que la Administración central había hecho muy poco por promover las lenguas y culturas diferentes del castellano, renunciando a promover una imagen plurinacional del Estado. Esta queja es especialmente intensa en relación a los medios: para Pujol, el Estado solo había mantenido en Cataluña el centro de producción de RTVE en Sant Cugat, que "ha ayudado muy poco»" ${ }^{75}$. Por eso, según el expresidente, la Generalitat tuvo que invertir tanto en este campo.

\section{CONCLUSIONES}

La información obtenida durante el trabajo de campo pone de manifiesto, con meridiana claridad, que Jordi Pujol siempre tuvo una concepción instrumental de los medios de comunicación, que entendía como herramientas para conseguir fines políticos diversos. Así lo indican tanto su comportamiento como también el pensamiento sobre el cual sustentó sus acciones.

Así, el comportamiento de Pujol coincide con la «instrumentalización» de los medios de comunicación que Hallin y Mancini consideran característica del sistema mediático Mediterráneo ${ }^{76}$. Esto se observa fácilmente en la entrada en la prensa durante el franquismo con la intención de promover su nacionalismo. El expresidente es bien claro reconociendo su voluntad de marcar la línea política de los medios de los cuales fue propietario como una manera de incidir en la transición a la democracia. A fin de cuentas, Pujol es explícito cuando reconoce que su apuesta personal por la inversión en medios de comunicación a partir de finales de los sesenta era para tomar posiciones dentro del nuevo escenario político que se abriría con la muerte de Franco y en el cual él quería jugar un papel destacado. En este sentido, el líder nacionalista entendió muy pronto la importancia de los medios en la política contemporánea y no estaba dispuesto a dejarlos solo en manos de los que consideraba sus contrincantes políticos.

En este sentido, las evidencias parecen indicar un doble objetivo ideológico por parte de Pujol. Por una parte, divulgar el catalanismo — del cual también participaban en grados diversos otros partidos como el PSC, PSUC o ERC - en un sentido amplio, no partidista. Esto aparece claramente expresado en el argumentario legitimador de la creación de la CCRTV. Por otra parte, la defensa de su ideario personal y de la acción de gobierno de su

75 Comunicación personal, 5 de septiembre de 2013.

76 Hallin y Mancini (2004). 
partido. La propiedad de El Correo Catalán hasta 1986 y el proyecto multimedia impulsado desde la Generalitat alrededor de este periódico y las subvenciones a medios más o menos afines y/o influyentes son las principales evidencias.

Desde este punto de vista, tanto la actitud como la acción de Pujol encajan en las previsiones teóricas de Guibernau sobre las necesidades de acceso a los medios específicas de los nacionalismos de oposición en los Estados nación contemporáneos ${ }^{77}$. De nuevo, el principal indicador es su entrada en la prensa a partir de 1968, cuando el régimen franquista reprimía al catalanismo y este pugnaba por hacerse oír. Pero también aparece con claridad cuando Pujol se queja del trato que el nacionalismo (y en concreto, su partido y sus Gobiernos) recibía tanto de la prensa barcelonesa durante la Transición como de los medios editados en Madrid durante todo su mandato. Sería esta lectura del escenario mediático lo que le habría convencido de la necesidad de crear medios catalanistas, tanto en el sentido amplio como en el partidista apuntado anteriormente. De acuerdo con Guibernau, pues, Pujol usó los medios para compensar la mala imagen que otros medios daban del movimiento político que promovía.

Ahora bien, tal y como sugerían las aportaciones de Gifreu y Guibernau, el papel que Pujol atribuyó a los medios de comunicación iba más allá de la instrumentalización tal y como lo definen Hallin y Mancini ${ }^{78}$. Para Pujol, los medios eran herramientas fundamentales para desplegar no solo su ideología, sino también su proyecto de "Construir Catalunya». En este punto hay tres evidencias claras. En primer lugar, el papel central atribuido a los medios para normalizar la situación del catalán y estimular su uso social. Por otra parte, la importancia otorgada a los medios para diseminar entre los habitantes de Cataluña un sentimiento de pertenencia y adhesión a la nación catalana. Finalmente, la concepción de los medios como instrumento de poder real, capaz de influir en la evolución de la sociedad, y por lo tanto elemento clave para el ejercicio efectivo del autogobierno. En este punto, el acceso de Pujol a la presidencia marcó claramente un antes y un después: las competencias de que disponía la Generalitat la convertían en un "casi-Estado» con una capacidad notable de intervención sobre la estructura y dinámica del sector mediático.

De acuerdo con los resultados obtenidos, pues, Pujol convirtió la creación de medios de comunicación en un objetivo político, entendiendo que eran fundamentales para desarrollar su proyecto "Construir Catalunya». Una vez existieron, fueron instrumentos que iban mucho más allá de la movilización

\footnotetext{
77 Guibernau (1999).

78 Gifreu (1991); Guibernau (2002), y Hallin y Mancini (2004).
} 
política o la difusión de ideología. Sin duda alguna, la normalización lingüística o la promoción de la identidad nacional no entran dentro de las previsiones teóricas de Hallin y Mancini, aunque sea cierto que son acciones habitualmente encomendadas por los actores políticos a los medios, tal como señala Guibernau ${ }^{79}$.

Las estrategias seguidas para conseguir los objetivos apuntados fueron cuatro, según las evidencias recogidas. En primer lugar, la inversión en medios impresos privados. Esta fue la primera estrategia que desarrolló, sencillamente porque era la única posible bajo el franquismo. La segunda estrategia, ya instalado en el Gobierno de la Generalitat, fue el empleo de las competencias autonómicas - en concreto, la concesión de frecuencias de FM- para crear medios privados autonómicos y configurar un grupo multimedia alrededor de la prensa adquirida durante la dictadura. La tercera fue la creación de la CCRTV y su constante crecimiento. Finalmente, la instauración de un sistema de subvenciones a la difusión de publicaciones periódicas $-\mathrm{y}$ otras de menos transparentes- cerraba el círculo. Como apunta Amat, los organismos parapolíticos (Banca Catalana y el CIRP) y la presidencia de la Generalitat eran instrumentos para sus objetivos nacionalizadores. Así, aquello que hasta la llegada a la Generalitat se llevaba a cabo con fondos privados, a partir de 1980 se aborda con todos los recursos de los que disponía la administración catalana, que no son solo económicos - aunque estos serían centrales incluso más allá de los años considerados aquí-.

Como se puede observar, se actuó sobre todos los medios existentes y legalmente posibles hasta 1988: prensa privada, radio pública y privada; y televisión pública. Sin duda, el proyecto era globalizante y aspiraba a construir un sistema mediático basado en actores autóctonos potentes prácticamente inexistente a finales de los setenta, tal como sugería Gifreu (1991). Una muestra más de las grandes dimensiones que alcanzó la intervención de Pujol sobre los medios de comunicación.

\section{Bibliografía}

Amat, J. (2015). El llarg procés. Cultura i política a la Catalunya contemporània (1937-2014). Barcelona: Tusquets Editors.

Antich, J. (1994). El Virrei. Barcelona: Planeta.

Baget, J. M. (1999). Quaranta anys de televisió a Catalunya (1959-1999). Barcelona: Enciclopèdia Catalana.

79 Hallin y Mancini (2004) y (1999). 
Barrera, C. (1995). Sin mordaza: veinte años de prensa en democracia. Madrid: Temas de Hoy. Bonet, M. (1994). Només una ràdio en català. Capçalera, 49, 11-18.

Carreras, Ll. (1987). La ràdio i la televisió a Catalunya, avui. Barcelona: Edicions 62.

Corbella, J. M. (1991). La comunicació social a Catalunya, 1981-1991. Una dècada de canvis. Barcelona: CEDIC.

Corominas, M. (1997). Estructura y polítiques de la comunicació. En J. Berrio (ed.). Un segle de recerca en comunicació a Catalunya. Estudi critic dels principals àmbits d'investigació de la comunicació de massa (pp. 99-150). Bellaterra: Servei de Publicacions de la Universitat Autònoma de Barcelona.

— y Moragas, M. (eds.) (2003). Informe de la comunicació a Catalunya 2001-2002. Bellaterra, Castelló de la Plana, Barcelona, València; Servei de Publicacions de la Universitat Autònoma de Barcelona; Publicacions de la Universitat Jaume I; Universitat Pompeu Fabra; Universitat de València, Servei de Publicacions.

Crameri, K. (2008). Catalonia: National identity and cultural policy 1980-2003. Cardiff: University of Wales Press.

Culla, J. B. (coord.) (2001). El pal de paller. Convergència democràtica de Catalunya (1974 -2000). Barcelona: Pòrtic.

Departament de Cultura (1985). Memòria del Departament de Cultura (gener-desembre 1983). Barcelona: Generalitat de Catalunya.

- (1987). Memòria del Departament de Cultura (1984-1985). Barcelona: Generalitat de Catalunya.

Dowling, A. (2013). La reconstrucció nacional de Catalunya (1939-2012). Barcelona: Pasado y Presente.

Favà, M. (2017). Diari Avui, 1976-2009. Entre el somni i l'agonia. Barcelona: Meteora.

Faulí, J., Riera, I., Huertas, J. M., Dalfó, X., Simó, I-C., Lanao, P. y Vinyoles, C. (1987). Tele/Estel, Arreu, Oriflama, Canigó i Presència. Barcelona: Diputació de Barcelona/ Col-legi de Periodistes de Catalunya.

Fernández, I. y Blasco, J. J. (2005). Las ayudas a la prensa en Cataluña (2000-2003). Sphera Pública, 5, 181-199.

Franquet, R. (2001). Història de la ràdio a Catalunya al segle XX. De la ràdio galena a la ràdio digital. Barcelona: Generalitat de Catalunya.

Geli, C. y Huertas, J. M. (1990). Les tres vides de Destino. Barcelona: Diputació de Barcelona/Col-legi de Periodistes de Catalunya.

Gifreu, J. (dir.) (1991). Construir l'espai català de comunicació. Barcelona: Cedic.

Guibernau, M. (1999). Nacions sense estat: nacionalisme i diversitat a l'era global. Barcelona: Columna.

- (2002). Nacionalisme català. Franquisme, transició i democràcia. Barcelona: Portic.

— (2010). La identitat de les nacions. Barcelona: Dèria.

Guillamet, J. (1996). Premsa, franquisme i autonomia. Crònica catalana de mig segle llarg (1939-1995). Barcelona: Flor del Viento.

Guimerà, J. À. (2002). Elements teòrics i metodològics per a l'estudi de l'estructura i les politiques de comunicació local a Catalunya (1979-2002). Bellaterra: Universitat Autònoma de Barcelona. 
- (2013). Polítiques de comunicació a Catalunya: una qüestió d'estat en revisió. En M. Civil, J. J. Blasco, J. À. Guimerà, (coords.). Informe de la comunicació a Catalunya 2011-2012 (pp. 257-268). Barcelona: Generalitat de Catalunya.

- (2014). Les politiques de comunicación durant els governs de Jordi Pujol. Premsa, ràdio i televisió en el procés de construcción nacional. Barcelona: Proa.

Hallin, D. C. y Mancini, P. (2004). Sistemas mediáticos comparados. Barcelona: Editorial Hacer.

Huertas, J. M. (2005). El plat de llenties. Periodisme i transició a Catalunya (1975-1985). Barcelona: Col-legi de Periodistes de Catalunya.

Jones, D. (1998). Investigación sobre comunicación en España: evolución y perspectivas. Zer, 5, 13-51.

- (2007). Pujol y la construcción de un espacio catalán de comunicación: interacciones entre instituciones políticas y empresas mediáticas. Ámbitos, 15, 499-524.

Lo Cascio, P. (2008). Nacionalisme i autogovern. Catalunya 1980-2003. Afers: Catarroja.

- Q Quevedo, C., Mayol, D. y Santacana, C. (2005). Els portaveus durant el franquisme. En J. Casassas (coord.). Premsa cultural i intervenció politica dels intel.lectuals a la Catalunya contemporània (1814-1975) (pp. 115-141). Barcelona: Publicacions i Edicions de la Universitat de Barcelona.

Martínez, F. y Oliveres, J. (2005). Jordi Pujol: en nom de Catalunya. Barcelona: Random House Mondadori.

Mateo, R. (1990). Els ajuts de l'Estat a la premsa a l'Europa Occidental. Barcelona: Cedic. Prenafeta, Ll. (1999). L'ombra del poder. Barcelona: Planeta.

Pujol, J. (2007). Memòries (1930-1980). Barcelona: Proa.

- (2009). Memòries. Temps de construir (1980-1993). Barcelona: Proa.

- (2012). Memòries. De la bonança a un repte nou (1993-2011). Barcelona: Proa.

Quintà, A. (1983). El projecte de tercer canal de TV. Barcelona: Generalitat de Catalunya.

Saura, V. (1998). Carlins, capellans, cotoners i convergents. Història d'El Correo Catalán (1876-1985). Barcelona: Diputació de Barcelona/Col-legi de Periodistes de Catalunya.

Soriano, J. (2007). L'ofici de comunicòleg. Vic: Eumo. 